\title{
Tobacco Smoking Can Potentiate C-fiber Evoked Potentials in Human Brain
}

\author{
Takahiro Miyazaki*, ${ }^{*, 2}$, Xiaohong Wang ${ }^{1}$, Koji Inui $^{1,2}$, Edward F. Domino ${ }^{3}$ and Ryusuke Kakigi ${ }^{1,2}$ \\ ${ }^{I}$ Department of Integrative Physiology, National Institute for Physiological Sciences, Myodaiji-cho, Okazaki 444-8585, \\ Japan; ${ }^{2}$ Department of Physiological Sciences, School of life Sciences, The Graduate University for Advanced Studies, \\ Hayama, Kanagawa, Japan; ${ }^{3}$ Department of Pharmacology, University of Michigan, Ann Arbor, MI 48109-0632, USA
}

\begin{abstract}
The effects of smoking and nicotine on ultralate laser evoked potentials (LEPs), the EEG responses to C-fiber stimulation by a laser beam, were investigated in humans. Ultralate LEPs were repeatedly measured in two sessions, one after smoking, and the other in abstinence from smoking. The dominant frequency of the background EEG alpha activity, heart rate and venous plasma nicotine concentration were also measured. The peak-to-peak amplitude of the two major components (N2 and P2) of ultralate LEPs was significantly correlated both with the plasma nicotine concentration and with the background alpha frequency. The results suggest an arousal effect of nicotine on C-fiber mediated pain. The effect of nicotine on C-fiber LEPs was in the opposite direction of that on A $\delta$ fiber LEPs. The difference between $\mathrm{C}$ and A $\delta$ fibers might indicate a difference in effects of nicotine on first and second pain responses.
\end{abstract}

Keywords: Electroencephalography, pain, unmyelinated nerve fibers, tobacco smoking, event related potentials, nicotine.

\section{INTRODUCTION}

Numerous studies have explored effects of smoking and nicotine on event related potentials (ERPs) [1, 2], including selected ERP components [3] and the P300 [4]. Most of these studies reported increased amplitudes and/or decreased latencies supporting the hypothesis that nicotine generally enhances brain processing. On the contrary, we recently found that $\mathrm{A} \delta$ fiber pain-related laser evoked potentials (LEPs) were reduced by smoking (Miyazaki et al., submitted 2009). Since the degree of the amplitude reduction was in parallel with the plasma concentration of nicotine, the finding was in line with previous studies showing antinociceptive effects of nicotine in animals $[5,6]$ and humans $[7,8]$.

Noxious stimuli applied to the skin, however, activate two discrete nociceptive pathways. The sharp or first pain is mediated by thinly-myelinated A $\delta$ fibers while the dull or second pain by unmyelinated $\mathrm{C}$ fibers. The sharp pain is considered to facilitate avoidance behaviors while the dull pain is thought to be related to more prolonged pain as in inflammation. Since the peripheral conduction velocity is markedly different between the two fibers (10 to 20 versus 0.5 to $2.0 \mathrm{~m} / \mathrm{s}$ ), evoked potentials due to $\mathrm{A} \delta$ and C-fibers can be clearly differentiated [9]. Special stimulating paradigms enable one to activate C-fibers exclusively [10]. This is the first report on the effects of tobacco smoking and nicotine on pain-related brain activities after selective $\mathrm{C}$ fiber stimulation.

*Address correspondence to this author at the Department of Integrative Physiology, National Institute for Physiological Sciences, 38 Nishigo-naka, Myodaiji-cho, Okazaki 444-8585, Japan; Tel: +81-564-55-7810; Fax: +81564-52-7913; E-mail: miyazaki@nips.ac.jp

\section{MATERIALS AND METHODS}

\section{Subjects}

Ten healthy male volunteers who had a smoking habit participated in the study. Since no clear cortical responses were evoked by laser stimulation in two subjects, probably due to a small signal-to-noise ratio when recording ultralate LEPs, data from eight subjects aged 24 to 43 (mean \pm SD: $32.9 \pm 6.6$ ) years were used. The mean number of years that the subjects have smoked was $11.2 \pm 8.3$ years. The mean number of cigarettes they were smoking per day was $10.3 \pm$ 5.1. The subjects had no history of psychiatric or neurological disorders, or substance abuse, which were confirmed by the authors; psychiatrists (T.M. and K.I) and a neurologist (R.K.). The study was approved in advance by the Ethics Committee of the National Institute for Physiological Sciences, Okazaki, Japan. Written consent was obtained from all the subjects.

\section{Stimulation and Recordings}

A Tm:YAG laser (BLM1000S, Carl Baasel Lasertechnik, Starnberg, Germany) was used for noxious stimulation. The laser pulses were $2000 \mathrm{~nm}$ in wavelength, $1 \mathrm{~ms}$ in duration, and $3 \mathrm{~mm}$ in diameter. A low intensity laser beam was applied to a tiny area of subjects' skin using a thin aluminum plate with numerous fine holes as a spatial filter, to selectively stimulate unmyelinated $\mathrm{C}$ fibers without activating $A \delta$ fibers as described previously $[10,11]$. The beam was applied to the dorsum of the right hand between the first and second metacarpal bones. The stimulation intensity was adjusted to a level at which stimulation produced a clear pricking $\mathrm{A} \delta$ mediated pain without the spatial filter $(156.3 \pm 9.5 \mathrm{~mJ})$. The laser energy was kept constant throughout the experiment for each session of each 
subject. To avoid tissue damage, the irradiated points were moved slightly for each stimulus.

LEPs were recorded using a scalp electrode placed at $\mathrm{Cz}$ referred to the linked earlobes $(\mathrm{A} 1+\mathrm{A} 2)$ according to the International 10/20 system. The EEG signals were recorded with a bandpass filter of 0.1 to $100 \mathrm{~Hz}$ at a sampling rate of $1000 \mathrm{~Hz}$, and then digitally filtered with a $50 \mathrm{~Hz}$ low-pass filter. The window of analysis was from $100 \mathrm{~ms}$ before to $1200 \mathrm{~ms}$ after the stimulus onset, and the prestimulus period was used as the DC baseline. For the analysis of the dominant alpha frequency of background EEG activity, electrodes were placed on $\mathrm{O} 1$ and $\mathrm{O} 2$. The impedance of the electrodes was kept below $5 \mathrm{kohm}$. A pair of electrodes on the supra- and infra-orbit of the right eye was used to reject blink artifacts. An electrocardiogram was used to measure the heart rate, using a pair of disk electrodes placed on each forearm.

\section{Procedures}

The experiments were conducted in two sessions, Smoking and Control, on a separate day starting at either 9 am or $1 \mathrm{pm}$. The starting time was counterbalanced between subjects and between sessions. The procedures of the experiment were the same for the two sessions, except that subjects did not smoke a cigarette in the Control session. For each session, subjects were required to be abstinent from smoking for at least twelve hours before the experiment. To confirm this, $\mathrm{CO}$ concentration in end-tidal expired air was measured at first. Subjects were asked to exhale into an analyzer (Micro CO, Micro Medical Ltd., Rochester, UK). Subjects were seated in an armchair in a quiet electrically shielded room with temperature controlled at 24 to $26^{\circ} \mathrm{C}$. An indwelling catheter for collecting venous blood samples was placed in the left cephalic vein. There were five runs of recordings at different times: before smoking (Pre), and 5, 20, 35 and 60 minutes after smoking. Just before each run, venous blood was collected for measurements of plasma nicotine concentration (PNC), and an electrocardiogram and EEGs were recorded for at least $20 \mathrm{~s}$ for heart rate and alpha frequency calculation, respectively.

The peak frequency of the background alpha activity was determined using fast Fourier transform (FFT). In each run, twelve stimuli were applied and LEPs were recorded. The inter-stimulus interval was varied at random between 14 and 19 sec. In the Smoking session, subjects smoked a cigarette with $1.0 \mathrm{mg}$ of nicotine for five minutes just after Pre, and then the remaining four runs followed. The Control session was conducted 5 to 20 (mean 11.2) days after the Smoking session for each subject. The plasma nicotine concentrations were measured using gas chromatography-mass spectrometry. The method was similar to that used previously to analyze urinary cotinine concentrations [12] with the addition of a solid phase extraction step carried out on an MCX column (Water Corporation, Milford, MA). The MCX column was prepared and the sample eluted as described previously [13]. The blood samples were from the same subjects that participated in our previous study (Miyazaki et al., submitted 2009). Since the sensation that the subjects perceived after laser stimulation varied from a mild pressure to burning pain as we previously reported [11], we did not measure subjective pain ratings in this study.

\section{Data Analysis}

For each run of LEP recordings, five artifact-free epochs with a clear response were averaged. The peak latency of N2 and P2, and the peak-to-peak amplitude of the N2/P2 complex were calculated using time frames with latency periods of 650 to $1000 \mathrm{~ms}$ and 700 to $1150 \mathrm{~ms}$ for N2 and $\mathrm{P} 2$, respectively. The effects of Smoking (the Smoking session versus the Control session) and Run were assessed by two-way analysis of variance (ANOVA). Changes in the N2/P2 amplitude and in the frequency of the background alpha-band EEG activity relative to those of Pre were calculated. The correlations between the N2/P2 amplitude and dominant alpha frequency, and between the N2/P2 amplitude and PNC were assessed by a coefficient, $r$. Data were expressed by the mean \pm standard deviation.

\section{RESULTS}

The expiratory $\mathrm{CO}$ concentration was 0 to 6 (mean $\pm \mathrm{SD}$ : $2.9 \pm 1.0)$ and 0 to $9(3.5 \pm 0.9) \mathrm{ppm}$ for the Smoking and Control sessions, respectively, indicating the instruction to be abstinent from smoking was followed by the subjects. The PNC, mean heart rate and dominant frequency of the alpha activity for each run are listed in Table 1. The PNC, heart rate and alpha frequency for Pre were not different between the Control and Smoking sessions ( $p=0.20, p=0.69$ and $p$ $=0.20$, respectively, paired- $t$ test). In the Smoking session, the heart rate, alpha frequency and PNC increased $5 \mathrm{~min}$ after smoking and gradually decreased (Fig. 1). Results of a two-way (Smoking and Run) ANOVA indicated that Smoking was a significant factor to determine PNC $(F(1,70)$ $=33.6, p<0.0001)$, heart rate $(F(1,70)=12.3, p=0.0008)$ and background alpha frequency $(F(1,70)=17.4, p<$ 0.0001). A significant interaction between Smoking and Run was also found for all of PNC $(F(4,70)=4.6, p=0.0022)$, heart rate $(F(4,70)=2.52, p=0.049)$ and alpha frequency $(F(4,70)=2.7, p=0.04)$. The mean $\mathrm{PNC}$, heart rate and background alpha rhythm were largest for the 5 min run.

The N2 and P2 components of LEPs were observed at $775 \pm 66$ and $892 \pm 70 \mathrm{~ms}$ after the onset of stimulation, respectively (Fig. 2A). The peak latencies of the N2 and P2 components and the N2/P2 peak-to-peak amplitude are listed in Table 1. A two-way (Smoking and Run) ANOVA indicated that the N2 latency did not differ significantly between the two sessions ( $p=0.11$ ) or among five runs ( $p=$ $0.34)$. Smoking and Run were also not a significant factor to determine the P2 latency ( $p=0.20$ and 0.22 , respectively). On the other hand, a two-way ANOVA showed that the $\mathrm{N} 2 / \mathrm{P} 2$ amplitude was significantly different among five runs $(F(4,70)=5.1, p=0.0012)$, but not between the Control and Smoking sessions $(p=0.74)$. Grand-averages of ultralate LEPs across subjects for each run are shown in Fig. (2A).

As shown in Fig. (2B), the behavior of the N2/P2 amplitude over the Runs for the Smoking session was similar to that of the dominant alpha frequency. To support this, the N2/P2 amplitude had a significant positive correlation with the dominant frequency of the background alpha rhythm in the Smoking session $(r=0.44, p=0.0047$, Fig. 3B). This positive correlation was also found in the Control session $(r$ $=0.33, p=0.037$, Fig. 3A). Both the dominant alpha frequency $(r=0.58, p<0.0001)$ and N2/P2 amplitude $(r=$ 
Table 1. The plasma Nicotine Concentration, Heart Rate, Dominant Alpha Frequency, N2 and P2 Latencies and N2/P2 Peak-toPeak Amplitude for Each Session

\begin{tabular}{|c|c|c|c|c|c|c|c|}
\hline Session & $\begin{array}{c}\text { PNC } \\
(\mathrm{ng} / \mathrm{ml})\end{array}$ & $\begin{array}{c}\text { HR } \\
(/ \mathrm{min})\end{array}$ & $\begin{array}{c}\text { AF } \\
(\mathrm{Hz})\end{array}$ & $\begin{array}{l}\text { N2I } \\
\text { (ms) }\end{array}$ & $\begin{array}{l}\text { P2I } \\
\text { (ms) }\end{array}$ & \multicolumn{2}{|c|}{$\begin{array}{l}\text { NPa } \\
(\mu \mathrm{V})\end{array}$} \\
\hline Pre & $2.5(1.2)$ & $66(8.7)$ & $10.0(0.3)$ & $750(52)$ & $875(76)$ & 21.8 & (4.3) \\
\hline $5 \mathrm{~min}$ & 2.6(1.4) & $63(7.4)$ & $10.0(0.3)$ & $760(70)$ & $876(75)$ & 19.7 & (3.9) \\
\hline $20 \mathrm{~min}$ & $2.5(1.1)$ & $60(6.5)$ & $10.1(0.3)$ & $767(84)$ & $883(85)$ & 20.7 & (4.6) \\
\hline $35 \min$ & $2.5(0.9)$ & $62(7.7)$ & $10.0(0.4)$ & $752(53)$ & $881(54)$ & 16.0 & (4.5) \\
\hline $60 \mathrm{~min}$ & $2.6(1.2)$ & $62(8.0)$ & $10.2(0.5)$ & 751(64) & $886(52)$ & 18.0 & (4.1) \\
\hline Smoking & $3.3(1.9)$ & $65(8.3)$ & $10.2(0.4)$ & $786(76)$ & $890(80)$ & 18.3 & (3.8) \\
\hline Pre & $23.5 \quad(17.7)$ & $79(10.8)$ & $11.1(0.8)$ & $813(100)$ & $947(105)$ & 20.3 & (3.3) \\
\hline $5 \mathrm{~min}$ & $11.1 \quad(8.3)$ & $70(9.5)$ & $10.5(0.5)$ & 789(44) & $881(51)$ & 19.0 & (5.8) \\
\hline $20 \min$ & $10.6 \quad(7.1)$ & $68(9.0)$ & $10.2(0.3)$ & $775(41)$ & $877(56)$ & 16.6 & (4.1) \\
\hline $35 \mathrm{~min}$ & $8.8(5.2)$ & $64(7.3)$ & $10.4(0.4)$ & $806(46)$ & $924(50)$ & 18.6 & (3.2) \\
\hline $60 \mathrm{~min}$ & & & & & & & \\
\hline
\end{tabular}

Data are given as mean (SD). PNC, plasma nicotine concentration; HR, heart rate; AF, alpha frequency; N2l, N2 latency; P2l, P2 latency; NPa, N2/P2 peak-to-peak amplitude.

$0.32, p=0.04$, Fig. 3C) were positively correlated with PNC in the Smoking session.

\section{DISCUSSION}

In the present study, a slight but significant facilitatory effect of smoking on C-fiber LEPs was found. The amplitude of LEPs was positively correlated with the plasma nicotine concentration (PNC). In the two-way ANOVA, a significant

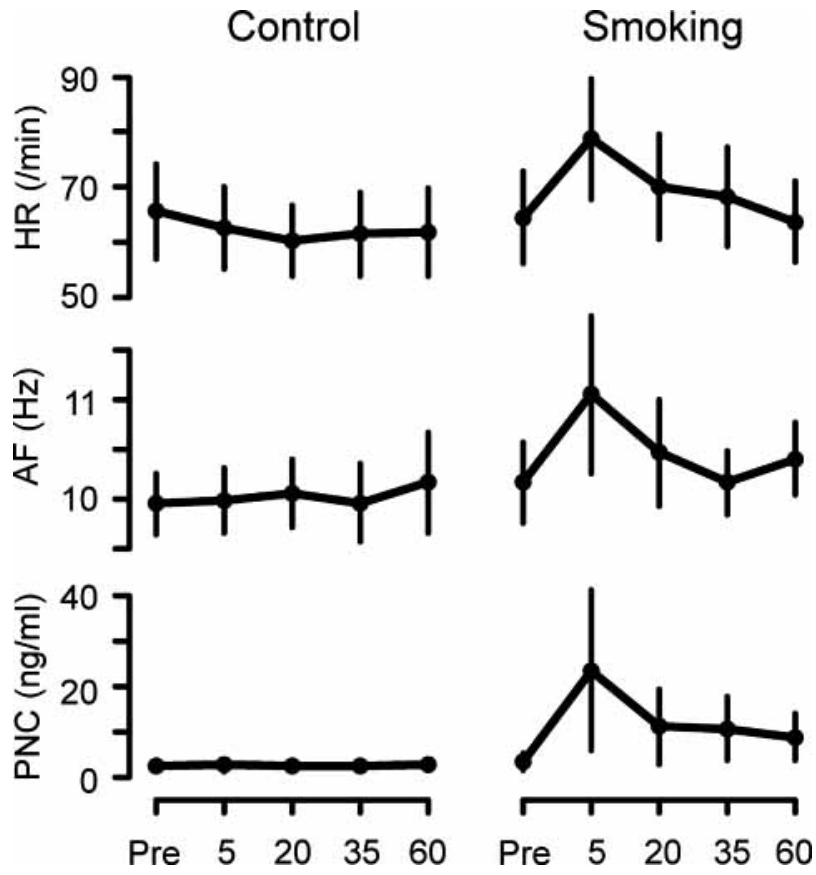

Fig. (1). Mean heart rate, dominant alpha frequency of the background EEG activity and plasma nicotine concentration for each run. The left and right columns correspond to the Control and Smoking sessions, respectively. Vertical bars show \pm SD. HR, heart rate; AF, dominant frequency of the background EEG activity; PNC, plasma nicotine concentration. main effect of Run was found on the N2/P2 peak-to-peak amplitude, although the effect of Session was not significant. These results indicate that the C-fiber LEPs were enhanced by nicotine intake but not changed by smoking itself. We previously reported an inhibitory effect of smoking on late

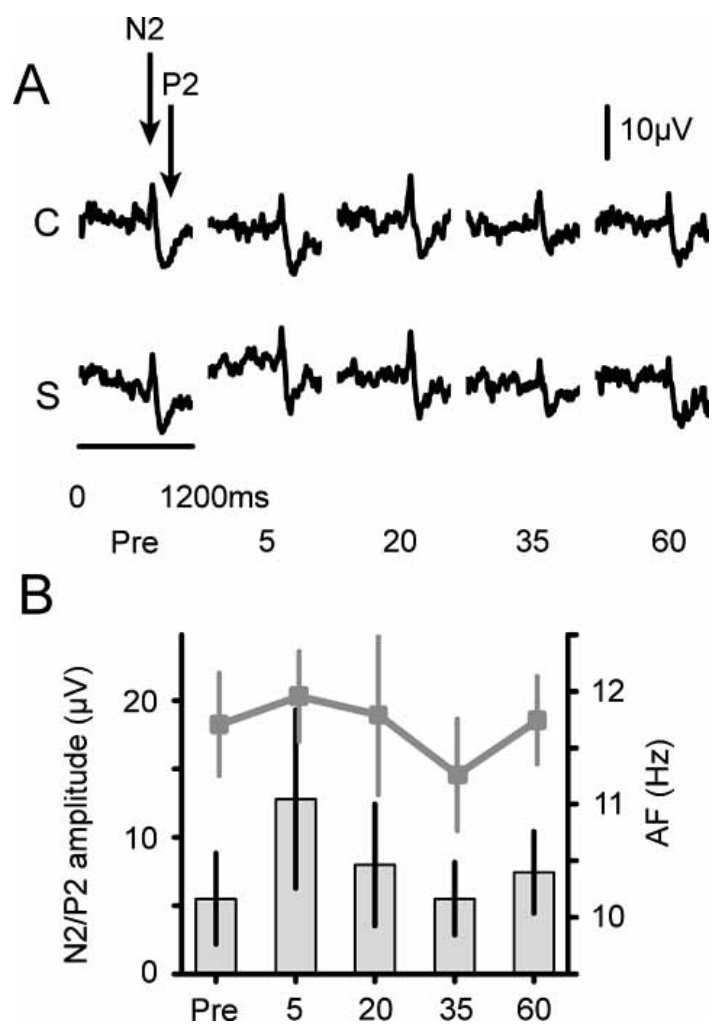

Fig. (2). Grand averaged waveforms of evoked potentials, and the N2/P2 amplitude and alpha frequency in the Smoking session. (A) Grand averaged waveforms for each run (Pre and 5, 20, 35 and 60 min) in the Control (C) and Smoking (S) sessions. (B) The N2/P2 amplitude (top) and dominant alpha frequency (bottom) for each run in the Smoking session. Vertical bars show \pm SD. 

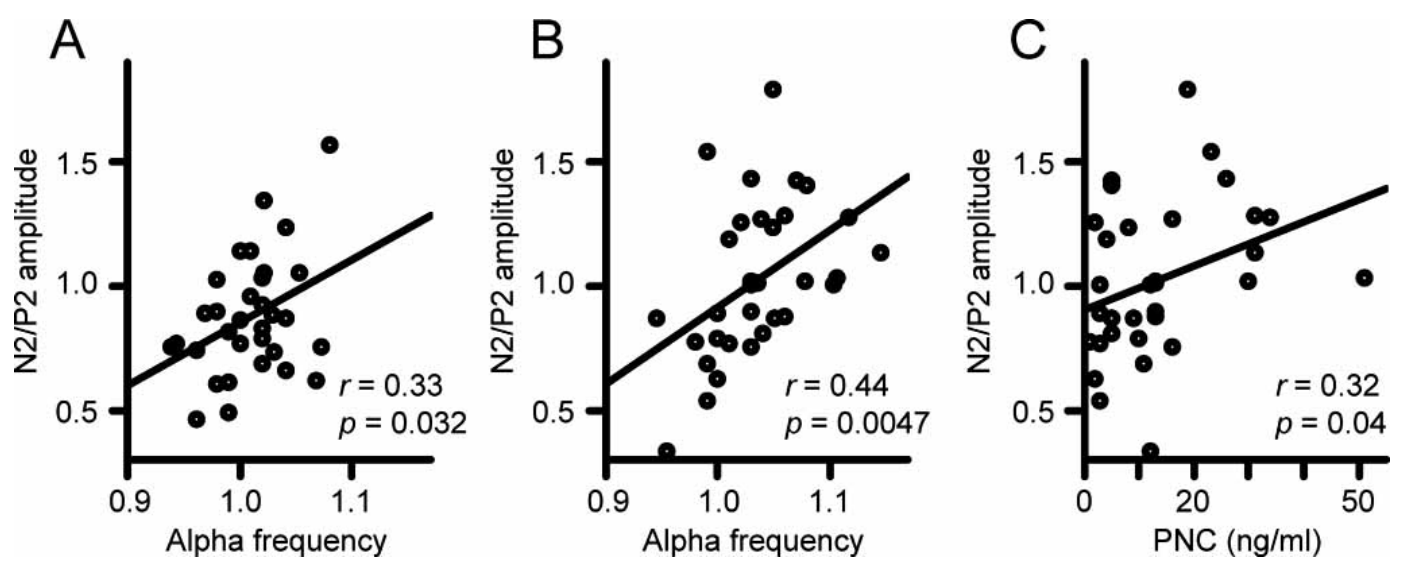

Fig. (3). Correlation of the N2/P2 amplitude with the dominant frequency of the background alpha activity and with the plasma nicotine concentration. A significant positive correlation was found between the N2/P2 amplitude and alpha frequency in the Control (A) and Smoking (B) sessions, and between the N2/P2 amplitude and plasma nicotine concentration in the Smoking session (C). Change from the baseline (Pre) was used for the N2/P2 amplitude and alpha frequency. AF, frequency of the background EEG activity; PNC, plasma nicotine concentration.

(A $\delta$ fiber) laser evoked potentials (Miyazaki et al., Submitted 2009). The P2 amplitude significantly decreased 5 minutes after smoking, and gradually increased. In addition, both the N2 and P2 amplitudes showed a significant negative correlation with PNC. In the present study, we examined whether tobacco smoking exerts a similar effect on ultralate (C-fiber) LEPs. Unexpectedly however, we found an effect of smoking on C-fiber LEPs in the opposite direction.

Most studies on effects of smoking and nicotine on EEG activities in humans support the hypothesis that smoking and nicotine have a facilitatory effect on the brain activity, by showing an increase in amplitude and/or a decrease in latency of evoked EEG components [1-4]. Many studies demonstrate that smoking and nicotine increase the dominant frequency of the alpha activity $[14,15]$. The positive effect of nicotine on the C-fiber LEP amplitude found in the present study is consistent with these studies. The N2/P2 amplitude was positively correlated with the dominant alpha frequency not only in the Smoking session but also in the Control session, where PNC remained at a very low level, suggesting that the change in the N2/P2 amplitude was affected by the change in vigilance. This idea is also supported by the finding by Qiu et al. [16] that the amplitude of ultralate LEPs was slightly increased during attention and significantly decreased during distraction, relative to control. In the present study, both changes of the N2/P2 amplitude and dominant alpha frequency followed the change in PNC after smoking. Taken together, the positive effect of nicotine on the C-fiber LEP amplitude in this study seems to reflect the general facilitatory effect of smoking and nicotine on brain activities.

The different effects of nicotine on the LEPs between the first pain ascending through the $\mathrm{A} \delta$ fibers and second pain through $\mathrm{C}$ fibers suggest a difference in the processing of these two types of pain. Several studies recently reported on the differences in cerebral processing between the first and second pain using EEG, magnetoencephalography (MEG) and functional magnetic resonance imaging (fMRI). In summary, EEG and MEG results show a similar main activation in the secondary somatosensory cortex after $\mathrm{A} \delta$ and $\mathrm{C}$ fiber stimulation except for the mean latency [10].
Activation in the primary somatosensory cortex following $\mathrm{A} \delta$ and $\mathrm{C}$ fiber activation is still a matter of controversy [1719]. By contrast, a clear difference was found using fMRI [20]. Brodmann's area 32/8/6 including the cingulate cortex and presupplementary motor area in the hemisphere ipsilateral to the stimulated side, and the bilateral anterior insula were significantly more activated following $\mathrm{C}$ fiber stimulation than A $\delta$ fiber stimulation. Since these areas are thought to be involved in the emotional aspect of pain processing, second pain perception might be more related to the emotional aspect than first pain perception. Tamura et al. $[21,22]$ reported that repetitive transcranial magnetic stimulation over the primary motor cortex increased the LEP amplitude and subjective pain rating scores following $\mathrm{A} \delta$ fiber stimulation but decreased them following $\mathrm{C}$ fiber stimulation. This difference could be attributed to a difference in the activity of the insular and cingulate cortices between the two conditions. Based on these studies in humans, it seems considerable that there was a difference in the effect of nicotine on first and second pain perception.

The present results may indicate the general arousal effect of nicotine in tobacco smoke, consistent with previous ERP studies. This effect might also have taken place in the first pain perception, but a larger antinociceptive effect on the first pain might have concealed it. Although the exact reason for this difference between $\mathrm{A} \delta$ and $\mathrm{C}$ fibers is unknown, the pharmacological and physiological effects of nicotine on the first and second pain are clearly different.

\section{CONCLUSION}

This is the first study to investigate the effect of tobacco smoking on pain-related brain activity after selective laser beam $C$ fiber stimulation. Smoking significantly increased the dominant alpha frequency. Both the N2/P2 amplitude of laser evoked potentials and dominant alpha frequency were positively correlated with the plasma nicotine concentration. Tobacco smoking/nicotine had the opposite effect on A $\delta$ fiber-mediated first pain and $\mathrm{C}$ fiber-mediated second pain. These differences may be important in understanding the actions of nicotine on the first and second pain responses. 


\section{ACKNOWLEDGMENT}

The nicotine assays were done by Dr. Linda von Weymarn and the Analytical Biochemistry Core at the Masonic Center, University of Minnesota Minneapolis, MN 55455, and supported by National Institute on Drug Abuse Grant DA 018974-01-03. This work was supported by a grant from the Smoking Research Foundation, Japan. The entire study has been conducted in a completely scientifically objective fashion. The authors caution that these research findings should not be interpreted as indicating tobacco smoking has any significant properties. Instead, the findings support basic scientific studies on the actions of nicotine in the brain.

\section{REFERENCES}

[1] Knott VJ, Hooper C, Lusk-Mikkelsen S, Kerr C. Cigarette smoking and event-related brain electrical potential (ERP) topographies associated with attentional-distractive processes. In: Domino EF, Ed. Brain imaging of nicotine and tobacco smoking. Ann Arbor: NPP Books 1995; pp. 191-221.

[2] Pritchard W, Sokhadze E, Houlihan M. Effects of nicotine and smoking on event-related potentials: a review. Nicotine Tob Res 2004; 6(6): 961-84.

[3] Woodson PP, Baettig K, Etkin MW, et al. Effects of nicotine on the visual evoked response. Pharmacol Biochem Behav 1982; 17(5): 915-20.

[4] Houlihan ME, Pritchard WS, Robinson JH. Effects of smoking/nicotine on performance and event-related potentials during a short-term memory scanning task. Psychopharmacology (Berl) 2001; 156(4): 388-96.

[5] Mattila MJ, Ahtee L, Saarnivaara L. The analgesic and sedative effects of nicotine in white mice, rabbits and golden hamsters. Ann Med Exp Biol Fenn 1968; 46(1): 78-84.

[6] Simons CT, Cuellar JM, Moore JA, et al. Nicotinic receptor involvement in antinociception induced by exposure to cigarette smoke. Neurosci Lett 2005; 389(2): 71-6.

[7] Pomerleau OF, Turk DC, Fertig JB. The effects of cigarette smoking on pain and anxiety. Addict Behav 1984; 9(3): 265-71.

[8] Perkins KA, Grobe JE, Stiller RL, et al. Effects of Nicotine on Thermal Pain Detection in Humans. Exp Clin Psychopharmacol 1994; 2(1): 95-106.
[9] Bromm B, Neitzel H, Tecklenburg A, Treede RD. Evoked cerebral potential correlates of C-fibre activity in man. Neurosci Lett 1983 43(1): 109-14

[10] Kakigi R, Tran TD, Qiu Y, et al. Cerebral responses following stimulation of unmyelinated C-fibers in humans: electro- and magneto-encephalographic study. Neurosci Res 2003; 45(3): 25575 .

[11] Tran TD, Lam K, Hoshiyama M, Kakigi R. A new method for measuring the conduction velocities of Abeta-, Adelta- and Cfibers following electric and $\mathrm{CO}(2)$ laser stimulation in humans. Neurosci Lett 2001; 301(3): 187-90.

[12] Hecht SS, Carmella SG, Chen M, et al. Quantitation of urinary metabolites of a tobacco-specific lung carcinogen after smoking cessation. Cancer Res 1999; 59(3): 590-6.

[13] Murphy SE, Villalta P, Ho SW, von Weymarn LB. Analysis of [3', $\left.3^{\prime}-\mathrm{d}(2)\right]$-nicotine and [3', $\left.3^{\prime}-\mathrm{d}(2)\right]$-cotinine by capillary liquid chromatography-electrospray tandem mass spectrometry. J Chromatogr B Anal Technol Biomed Life Sci 2007; 857(1): 1-8.

[14] Knott VJ. Dynamic EEG changes during cigarette smoking. Neuropsychobiology 1988; 19(1): 54-60.

[15] Michel C, Hasenfratz M, Nil R, Bättig K. Cardiovascular, electrocortical, and behavioral effects of nicotine chewing gum Klin Wochenschr 1988; 66 (Suppl 11): 72-9.

[16] Qiu Y, Inui K, Wang X, Tran TD, Kakigi R. Effects of attention, distraction and sleep on $\mathrm{CO}(2)$ laser evoked potentials related to $\mathrm{C}$ fibers in humans. Clin Neurophysiol 2002; 113(10): 1579-85.

[17] Forss N, Raij TT, Seppä M, Hari R. Common cortical network for first and second pain. Neuroimage 2005; 24(1): 132-42.

[18] Ploner M, Gross J, Timmermann L, Schnitzler A. Cortical representation of first and second pain sensation in humans. Proc Natl Acad Sci USA 2002; 99(19): 12444-8.

[19] Tran TD, Inui K, Hoshiyama M, Lam K, Qiu Y, Kakigi R. Cerebral activation by the signals ascending through unmyelinated $\mathrm{C}$-fibers in humans: a magnetoencephalographic study. Neuroscience 2002; 113(2): 375-86.

[20] Qiu Y, Noguchi Y, Honda M, et al. Brain processing of the signals ascending through unmyelinated $\mathrm{C}$ fibers in humans: an eventrelated functional magnetic resonance imaging study. Cereb Cortex 2006; 16(9): 1289-95.

[21] Tamura Y, Hoshiyama M, Inui K, et al. Facilitation of A[delta]fiber-mediated acute pain by repetitive transcranial magnetic stimulation. Neurology 2004; 62(12): 2176-81.

[22] Tamura Y, Okabe S, Ohnishi T, et al. Effects of 1-Hz repetitive transcranial magnetic stimulation on acute pain induced by capsaicin. Pain 2004; 107(1-2): 107-15. 